\title{
Aplicación del MEB-EDS en el estudio morfológico y químico-elemental de hidroxiapatita sintetizada en presencia de PVP
}

\author{
Application of the SEM-EDS on the morphological and chemical-elemental studies of \\ the synthesized hydroxyapatite with PVP

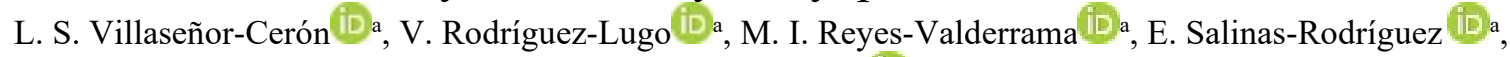 \\ D. Mendoza-Anaya $1 \mathrm{D}$ b,*
}

a Área Académica de Ciencia de la Tierra y Materiales, Universidad Autónoma del Estado de Hidalgo, 42184, Pachuca, Hidalgo, México.

${ }^{b}$ Instituto Nacional de Investigaciones Nucleares, Carr. México-Toluca S/N, La Marquesa, C.P. 52750 Ocoyoacac, Edo. De México, México.

\section{Resumen}

En este trabajo se presentan los resultados obtenidos en el estudio morfológico y químico-elemental, a través de MEB-EDS, de la hidroxiapatita sintetizada por el método hidrotermal asistido por microondas utilizando diferentes concentraciones de polivinilpirrolidona (PVP) como controlador de tamaño y forma de las partículas de Hap. De acuerdo a lo observado con el MEB la presencia de PVP durante la síntesis de la hidroxiapatita facilita el crecimiento de nanoestructuras tipo rods, observando un aumento de la longitud cuando la cantidad de PVP adicionada en la síntesis se incrementaba. El análisis por EDS mostró la presencia de oxígeno, calcio y fósforo como elementos mayoritarios en la Hap pura y sintetizada en presencia de PVP; también indicó que para una concentración de PVP de $0.3 \%$ en peso se obtiene una proporción $\mathrm{Ca} / \mathrm{P}$ de 1.69 , próxima a la estequiométrica. Tomando en cuenta que muchas de las propiedades de la Hap dependen de la escala dimensional en la que se realiza el estudio, la información que se obtuvo a través del MEB-EDS en la escala micro y nanoestructural de la hidroxiapatita es científica y tecnológicamente relevante; por tal razón, en este trabajo se incluye una breve explicación del principio de operación del MEB-EDS.

Palabras Clave:

Hidroxiapatita, Método Hidrotermal, Polivinilpirrolidona, Controlador de tamaño, MEB, EDS.

\begin{abstract}
This work presents the results obtained on the morphological and chemical-elemental studies, using SEM-EDS, of the hydroxyapatite synthesized by the microwave assisted hydrothermal method, in which, several concentrations of Polyvinylpyrrolidone (PVP) was used as controller of the size and shape of the Hap particles. According to the SEM results, the PVP promotes the growth of the nanorods structures, where the length was growing when the concentration added of PVP was increasing. The EDS analysis shows that oxygen, calcium and phosphorous were the main elements present in the different Hap samples; it was also observed that Hap sample synthesized in presence of $0.3 \mathrm{wt} \%$ of PVP, the rate of $\mathrm{Ca} / \mathrm{P}$ was 1.69 , which is near to the stoichiometric. Finally, these results showed that SEM-EDS is a valuable tool to the study of hydroxyapatite, for this reason a brief introduction of the basic principles of the SEM-EDS operation was also included.
\end{abstract}

Keywords:

Hydroxyapatite, Hydrothermal method, Polyvinylpyrrolidone, Control size, SEM, EDS.

\section{Introducción}

La hidroxiapatita (Hap) es un biomaterial cerámico cuya importancia radica en que es el componente principal de huesos y dientes de mamíferos, incluyendo los humanos (Cerón, 2018; Wang, 2010; Sánchez-Campos, 2020 y 2021; Zhang, 2007;
López-Ortiz, 2020; Rodríguez-Lugo, 2018 y 2020). Este biomaterial ha sido sintetizado y analizado con el principal objetivo de ser utilizado para regenerar el hueso, que por fracturas o alguna enfermedad degenerativa, ha sufrido algún daño. Por sus propiedades fisicoquímicas de porosidad y adsorción, la Hap también es utilizada como sensor de gases y para atrapar iones pesados contaminantes (Villaseñor, 2019; Montes de Oca, 2019).

\footnotetext{
*Autor para la correspondencia: demetrio.mendoza@inin.gob.mx

Correo electrónico: leslysabina@gmail.com (Lesly Sabina Villaseñor-Cerón), ventura.rl65@gmail.com (Ventura Rodríguez-Lugo), isareyv@hotmail.com (María Isabel ReyesValderrama), salinasr@uaeh.edu.mx (Eleazar Salinas Rodríguez), demetrio.mendoza@inin.gob.mx (Demetrio Mendoza Anaya). 
Previo a una aplicación real, la Hap debe ser completamente caracterizada para conocer su morfología (tamaño y forma de las partículas constituyentes) y composición elemental, además de otras características, por ejemplo, cristalográficas; este estudio permite evitar un comportamiento adverso en su aplicación y así incrementar su eficiencia.

La forma y tamaño de las partículas de hidroxiapatita son parámetros fundamentales que influyen drásticamente en las características y propiedades físico-químicas de ésta, y por lo tanto, en el éxito durante su aplicación (López-Ortiz, 2020; Sánchez-Campos, 2021; Rodríguez-Lugo, 2018). Por tal razón, el control del tamaño y la forma de la partícula durante la síntesis es primordial; para lograrlo se pueden seguir rutas de síntesis donde el tipo de reactivos, velocidad de reacción, $\mathrm{pH}$ o temperatura durante la síntesis, son variables a considerar para obtener como producto, hidroxiapatita con morfología específica. Sin embargo, el uso de ciertos surfactantes durante la síntesis, ha también permitido el control de la morfología de la hidroxiapatita, por lo que el objetivo del presente trabajo fue sintetizar hidroxiapatita, a través del método hidrotermal asistido por microondas, utilizando polivinilpirrolidona o simplemente PVP $\left[\left(\mathrm{C}_{6} \mathrm{H}_{9} \mathrm{NO}\right) \mathrm{n}\right]$ para controlar la morfología de la Hap. Se debe mencionar que este polímero tiene $\mathrm{C}=\mathrm{O}, \mathrm{C}-\mathrm{N}$ y $\mathrm{CH}_{2}$ como grupos funcionales y es ampliamente utilizado como controlador de forma y tamaño durante la síntesis de nanopartículas metálicas, por ejemplo, de $\mathrm{Au}, \mathrm{Ag}$ y Cu (Kallum, 2015; Li, 2015), pero su uso en la síntesis de Hap para controlar tamaño y forma ha sido menos estudiada (Nathanael, 2015), aunque si para la síntesis de biomateriales compuestos Hap-polímero (Gła, 2021).

En la caracterización de la Hap generalmente se utilizan diferentes técnicas analíticas, tales como el microscopio electrónico de barrido (MEB) acoplado con espectroscopia por dispersión de energía de rayos $\mathrm{X}$ (EDS). El MEB es una herramienta útil con la que es posible obtener información sobre la microestructura de la Hap, hábito de crecimiento de las partículas, porosidad y composición químico-elemental (VernonParry, 2000; Ipohorski, 2013; Ledea, 2010; Mohammed, 2018). Con el fin de complementar este trabajo, también se presenta una breve explicación del principio de operación del MEB y su aplicación en la caracterización de la Hap.

En el MEB (Figura 1), un haz de electrones emitidos por un cátodo o filamento de tungsteno que es calentado a aproximadamente $2700{ }^{\circ} \mathrm{C}$ (por un proceso de emisión termoiónica) pasa a través de una columna en la que se ha hecho un vacío de alrededor de $10^{-6} \mathrm{~Pa}$, al ser atraídos por el ánodo, mantenido a un voltaje positivo (puede ser entre 0.5 y $30 \mathrm{KV}$ ) respecto al cátodo; a esta diferencia de potencial entre el cátodo y el ánodo se le conoce como voltaje de aceleración (el MEB trabaja comúnmente con voltajes desde $5 \mathrm{KV}$ hasta $30 \mathrm{KV}$ ). Es importante resaltar que el filamento puede ser también de hexaboruro de lantano y de emisión de campo (González Mancera, 2006), aunque el proceso de emisión es diferente, generan un haz más coherente, sin embargo, el costo económico es más alto.

Al continuar su paso el haz de electrones a través de esta columna, el diámetro del haz inicial es concentrado por una serie de lentes electromagnéticas: condensadora y objetiva (Figura 1) hasta alcanzar unos nanómetros $(\approx 1 \mathrm{O} \mathrm{nm})$; es decir, su diámetro va disminuyendo hasta hacerse casi puntual. De manera simultánea, la intensidad de corriente disminuye hasta en 2 órdenes de magnitud $(\approx \mu \mathrm{A})$. Cabe decir, que las lentes consisten en bobinas o solenoides enrollados por las que se hace pasar una corriente eléctrica generándose un campo magnético; de esta manera, a través de fuerzas electromagnéticas los electrones son dirigidos a un punto específico.
El haz electrónico ya colimado o puntual, es desplazado sobre toda la superficie predeterminada de la muestra a modo de un pincel que iría barriendo la superficie con continuas idas y venidas: esta movilidad del haz se consigue gracias a un sistema de bobinas de barrido situadas en la columna del microscopio (Figura 1). El diámetro final del haz (o spot size) influye en la resolución y depende de la capacidad que tenga el sistema ópticoelectromagnético (lentes) para disminuir el diámetro.

En resumen, el MEB es un instrumento que enfoca un haz de electrones a través de una serie de lentes magnéticas para interaccionar (o hacer incidir) en una zona (selecta) específica de la muestra en estudio y de allí formarse una imagen magnificada. En el proceso de interacción del haz con la superficie del material se generan diferentes señales: electrones secundarios, electrones retrodispersos y rayos $\mathrm{X}$ característicos; cada una extrae información específica del material con las que se forman imágenes asociadas con la morfología y composición elemental del material (Figura 1).

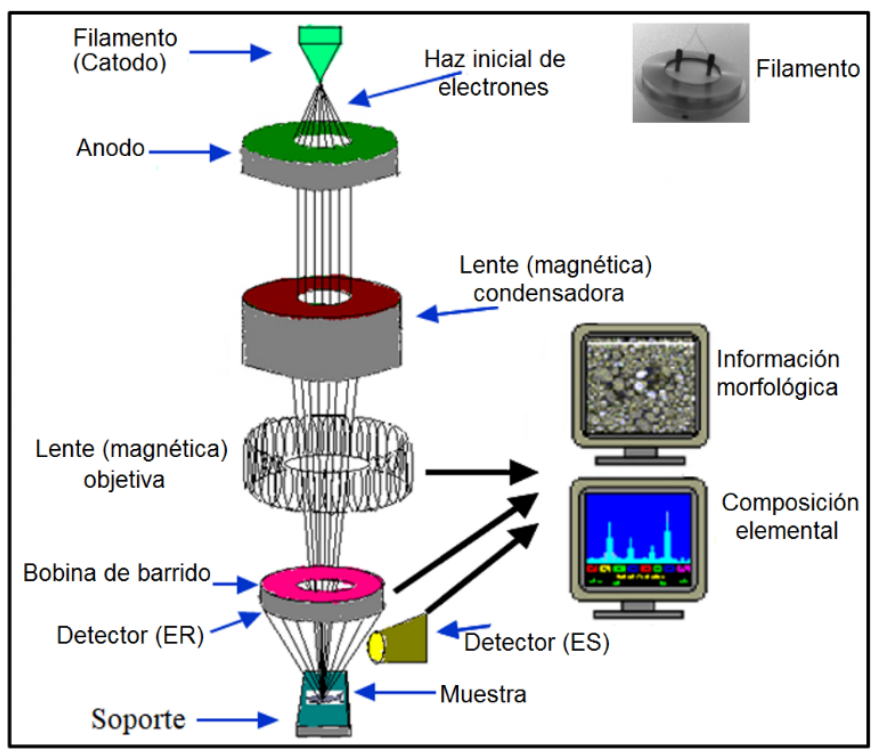

Figura 1. Esquema representativo del microscopio electrónico de barrido.

Al incidir el haz de electrones con la superficie de la muestra, el tamaño del volumen de interacción dependerá de la corriente del haz de electrones incidente, voltaje de aceleración y densidad atómica de la muestra. A mayor corriente y voltaje de aceleración, el haz penetrará la muestra a una mayor profundidad y, entre más elementos químicos con números atómicos grandes estén presentes, el haz penetrará menos la muestra. En la Figura 2 se presenta gráficamente la forma del volumen de interacción del haz y la profundidad a la que se generan las diferentes señales que son utilizadas para formar imágenes y análisis elemental; como se mencionó, la profundidad a la que se generan las diferentes señales depende de las condiciones de operación del MEB y de la naturaleza de la muestra. 


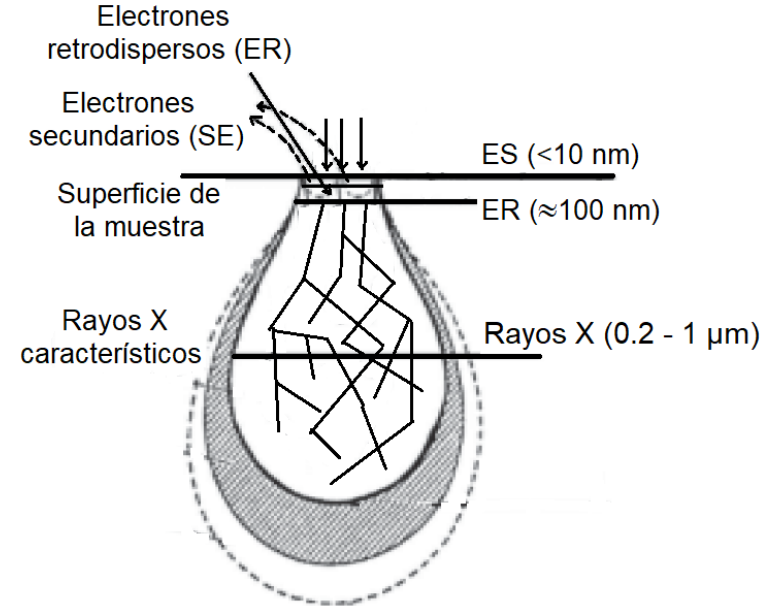

Figura 2. Esquema representativo del volumen de interacción del haz de electrones en la muestra.

Los electrones secundarios se producen cuando los electrones del haz primario interaccionan con los electrones de los átomos de la muestra, proporcionando la energía suficiente o necesaria para saltar fuera de las órbitas electrónicas; en este caso, el átomo queda positivamente ionizado. Los electrones expulsados son de muy baja energía (por debajo de $5 \mathrm{eV}$ ), por lo que sólo los que se emiten cerca de la superficie alcanzarán a llegar al detector y son considerados como electrones secundarios. Esta señal, en consecuencia lleva información principalmente del relieve o topografía de la muestra (Figura 3a).

La señal de electrones retrodispersos se produce cuando un electrón en el haz primario choca frontalmente con el núcleo de un átomo de la muestra, siendo repelido en sentido contrario fuera de la muestra. La intensidad de dicho efecto varía proporcionalmente con el número atómico de la muestra (Figura $3 b$ ); por ejemplo, átomos ligeros repelerán al haz incidente con menor energía y átomos pesados los repelerán con mayor energía. Por lo que la imagen formada por esta señal, brinda información principalmente sobre la distribución elemental en la zona analizada.

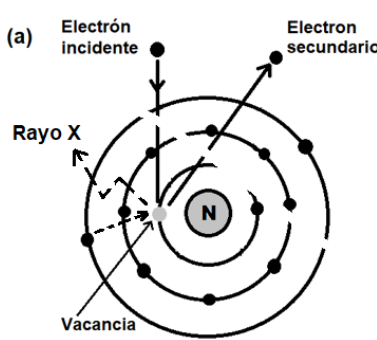

Átomo perteneciente a la muestra

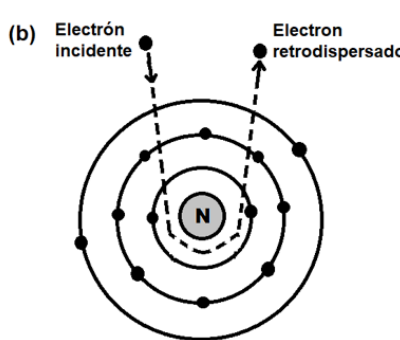

Átomo perteneciente a la muestra
Figura 3. (a) Generación de la señal de electrones secundarios $y$ rayos $X$ característicos, $y$ (b) electrones retrodispersos, ambos utilizados para formar imágenes en el MEB.

Cuando en el proceso de interacción del haz incidente con la muestra en estudio, ciertos átomos que constituyen la muestra son ionizados al desprenderse electrones de las capas electrónicas internas (Figura 3a), estos átomos presentan vacancias electrónicas quedando energéticamente inestables. Para alcanzar la estabilidad, electrones de capas electrónicas externas podrán llenar vacancias internas (Figura $3 \mathrm{a}$, flecha punteada); en este proceso, el átomo se queda con un remanente o excedente de energía que es liberada vía emisión de un rayo $\mathrm{X}$ (característico) (Figura 3a). La diferencia de energía que se libera, será mayor cuando la transición se da entre los orbitales K-L (rayo $X_{\mathrm{K} \alpha}$ ) o K-
$\mathrm{M}$ (rayo $X_{\mathrm{K} \beta}$ ), que cuando se da entre los orbitales L-M (rayo $X_{\mathrm{L} \alpha}$ ) ó M-N (rayo $X_{\mathrm{M} \alpha}$ ); debido a esto, los rayos $\mathrm{X}$ de las líneas $\mathrm{K}$ tienen generalmente una mayor energía, y en el espectro de energías se observa un pico de mayor intensidad (González Mancera, et.al., 2006). La energía de un rayos $X$ característico solo dependerá del átomo que lo emite, independientemente del estado químico en que se encuentre, y del orbital electrónico en que se da el evento (capa $\mathrm{K}, \mathrm{L}, \mathrm{M}$, etc.); por ejemplo, el carbono, oxígeno y aluminio emitirán rayos $\mathrm{X}_{\mathrm{K} \alpha}$ de $0.277,0.523$ y $1.486 \mathrm{KeV}$, respectivamente. Mientras que otros emitirán más de un solo rayos X, dependiendo de su configuración electrónica; por ejemplo, el hierro $(\mathrm{Fe})$ emite un rayo $\mathrm{X}_{\mathrm{K} \alpha}$ de $6.403 \mathrm{KeV}$ y un rayos $\mathrm{X}_{\mathrm{L} \alpha}$ de $0.705 \mathrm{KeV}$, en tanto que la plata $(\mathrm{Ag})$ emitirá rayos $\mathrm{X}$ asociados con las transiciones K-L, L-M y L-M; esto es, a medida que el número atómico y por lo tanto, la configuración electrónica se hace más compleja, la cantidad de rayos $\mathrm{X}$ característicos emitidos por el elemento, se incrementa también.

A excepción de los dos primeros elementos de la tabla periódica $(\mathrm{H}, \mathrm{He})$ que no emiten rayos $\mathrm{X}$ característicos y de los siguiente tres en la tabla $(\mathrm{Li}, \mathrm{Be}$ y $\mathrm{B})$ que emiten rayos $\mathrm{X}$ de baja energía para poder detectarse por los sistemas actuales, todos los demás elementos pueden ser identificados a través de los rayos $\mathrm{X}$ que emiten, y utilizados para conocer la composición elemental de la muestra en estudio e incluso hacer mapeos elementales que permitan conocer la distribución de estos elementos en la muestra; esto último se logra cuando la señal de rayos $X$ es utilizada para controlar intensidad de la imagen de barrido. La técnica que aprovecha este principio físico se conoce como espectroscopia por dispersión de energía de rayos X (EDS), la cual además de identificar los elementos presentes en la muestra, presenta un cálculo semicuantitativo de la concentración ( $\%$ en peso o \% atómico) de elementos identificados. Se debe mencionar que la cuantificación elemental depende de las condiciones de operación del microscopio (voltaje, spot size, distancia de trabajo), por lo que para un estudio comparativo, éste debe realizarse en las mismas condiciones.

La información que se obtiene a partir de la imagen formada con la señal de electrones secundarios y retrodispersos permite conocer las características micro-estructurales de un material; por ejemplo, morfología (tamaño y forma de las partículas) y porosidad, en escalas que incluyen los micrómetros y nanómetros (magnificaciones desde $\approx 10 \mathrm{X}$ hasta $\approx 300000 \mathrm{X}$ ), con imágenes (en escala de grises) con alto grado de profundidad. Además, es posible conocer la composición químico elemental para cada zona visualizada, siendo posible el análisis por área selecta, lineal, puntual y mapeo. En resumen y como ya se mencionó líneas arriba, la microscopia electrónica de barrido permitirá hacer un seguimiento detallado sobre la morfología y composición elemental de la Hap y el papel que juega el PVP como controlador de forma y tamaño durante la síntesis de la Hap.

\section{Procedimiento experimental}

La Hap pura se preparó con la disolución de la sal de $(\mathrm{Ca}$ $\left(\mathrm{NO}_{3}\right)_{2} 4 \mathrm{H}_{2} \mathrm{O}$ ) (Meyer) en $40 \mathrm{~mL}$ de agua destilada y agitación constante por 5 minutos; una vez disuelta la sal, se añadió directamente el $\left.\left(\mathrm{NH}_{4}\right)_{2} \mathrm{HPO}_{4}\right)$ (Meyer) a la solución al cabo de 15 min, el hidróxido de amonio $\left(\mathrm{NH}_{4} \mathrm{OH}\right)$ (Meyer) se agregó gota a gota a la solución, con la finalidad de aumentar el pH de 3.9 a 10. Posteriormente la solución se transfirió a un reactor de teflón para ser introducidas al microondas. Las condiciones de síntesis programadas fueron: a una potencia de $800 \mathrm{~W}$ por $10 \mathrm{~min}$ hasta alcanzar una temperatura de $200^{\circ} \mathrm{C}$ durante $30 \mathrm{~min}$. El sedimento obtenido fue lavado 3 veces consecutivamente con agua destilada 
y posteriormente fue secada a $100^{\circ} \mathrm{C}$ por $24 \mathrm{~h}$. Finalmente, el material fue molido hasta obtener un polvo fino y finalmente calcinado a $500^{\circ} \mathrm{C}$ por $3 \mathrm{~h}$. Para la síntesis utilizando el PVP (sigma aldrich) como controlador, este fue adicionado de manera diluida a la solución de $\left(\mathrm{Ca}\left(\mathrm{NO}_{3}\right)_{2} \quad 4 \mathrm{H}_{2} \mathrm{O}\right)$ y $\left.\left(\mathrm{NH}_{4}\right)_{2} \quad \mathrm{HPO}_{4}\right)$, continuando con un proceso similar para la síntesis de Hap pura. Se prepararon muestras de Hap con concentraciones de PVP de $0.1,0.3$ y $0.5 \%$ en peso $(\% \mathrm{wt})$.

La caracterización microestructural se realizó en un microscopio electrónico de barrido de presión controlada, marca Jeol, modelo JSM5900LV (resolución, $5 \mathrm{~nm}$ ), que tiene acoplada una sonda para análisis químico elemental, por EDS marca Oxford, modelo 7274 (resolución, $133 \mathrm{eV}$ ). El MEB operó en el modo de bajo vació (16 Pa) para el análisis elemental y en alto vacío $\left(\approx 10^{-6} \mathrm{~Pa}\right)$ para la obtención de imágenes, las cuales se obtuvieron con la señal de electrones secundarios principalmente. Previo, las muestras fueron montadas en portamuestras de aluminio y sujetas con cinta adhesiva de aluminio y recubiertas con $\mathrm{Au}$, por el método de sputtering; esto último, es para eliminar la acumulación de carga electrostática superficial en las muestras, durante el análisis.

\section{Resultados}

La Figura 4a muestra una micrografía donde se observa la morfología característica de la Hap pura, con agregados o cúmulos de partículas de diferente forma y tamaño. La Figura $4 \mathrm{~b}$ presenta una micrografía con mayor magnificación donde se aprecia que los agregados están constituidos por partículas nanométricas con aparente tendencia esférica.

La figura 5a presenta una imagen de la Hap que contiene 0.1 $\%$ wt de PVP. Al igual que en la Hap pura, se observan agregados de diferente forma y tamaño, además de algunas partículas micrométricas de superficie lisa o plana (señalado con una flecha). La Figura 5 b presenta una micrografía a con mayor amplificación donde se puede apreciar partículas nanométricas similares a las observadas en la Hap pura, aunque también se aprecia una partícula en forma de rod (señalada también con una flecha).

Las Figuras $6 \mathrm{a}$ y $6 \mathrm{~b}$ presentan las micrografías correspondientes a la muestra de Hap con 0.3 \%wt de PVP. A menor magnificación (Figura 6a) se observan agregados similares a los presentes en la Hap pura; sin embargo, también se aprecian partículas disgregadas con una apariencia tipo rods. A mayor magnificación (Figura 6b) es posible apreciar que los agregados se constituyen por partículas granulares de tamaño nanométrico.. También se observan estructuras tipo rods de anchos que van de decenas hasta cientos de nanómetros y longitudes que pueden rebasar el micrómetro. Cabe mencionar que en la síntesis de nanopartículas de Rh utilizando PVP como estabilizador, Ramírez-Meneses y colaboradores (2015) reportan fuertes aglomerados de nanopartículas debido a la presencia del polímero.

Las Figuras $7 \mathrm{a}$ y $8 \mathrm{~b}$ presentan las micrografías correspondientes a la Hap sintetizada adicionando $0.5 \%$ wt de PVP. En ambas es posible apreciar que las partículas presentes son estructuras tipo rods de anchos nanométricos más homogéneo respecto de la Hap con $0.3 \%$ wt de PVP. De manera similar, la longitud o largo de estas estructuras pueden alcanzar los micrómetros.
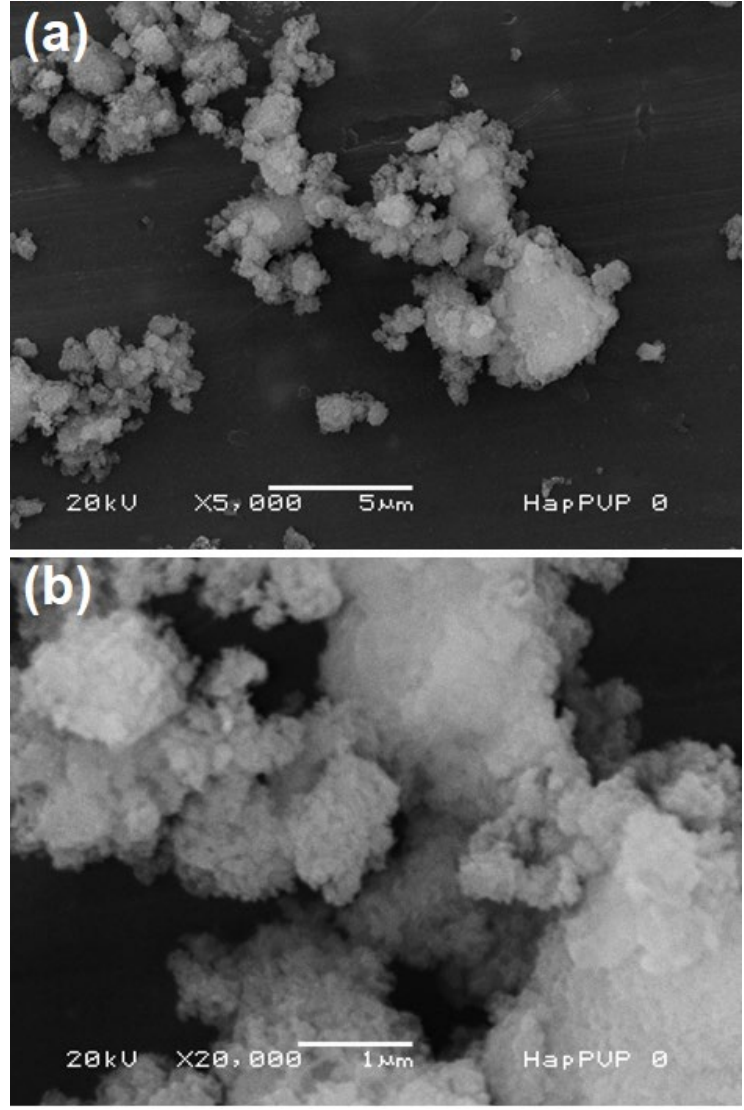

Figura 4. Micrografías a diferente magnificación pura.
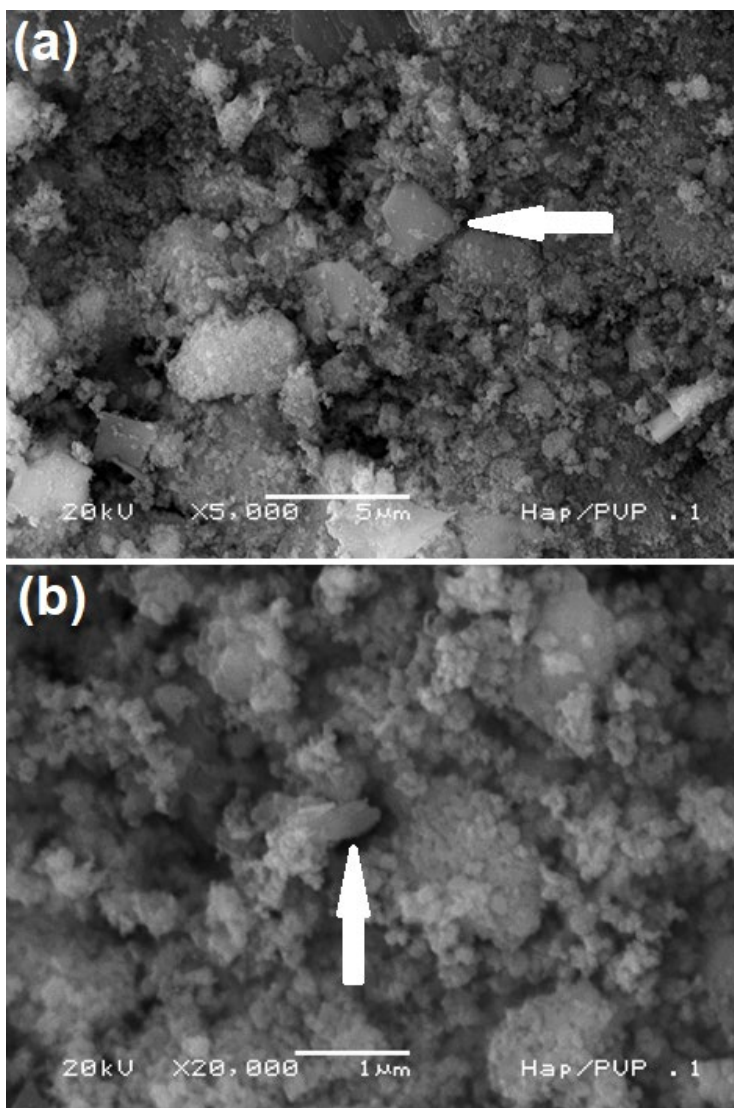

Figura 5. Micrografías a diferente magnificación donde se aprecia la morfología característica de Hap sintetizada con 0.1 \%wt de PVP. 

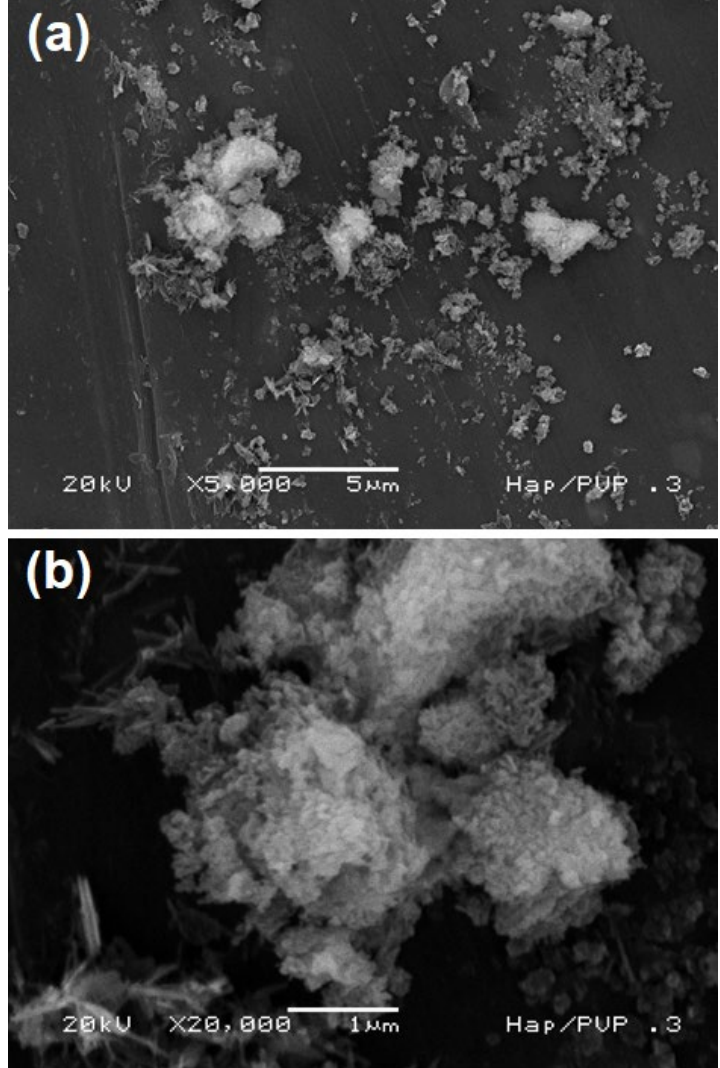

Figura 6. Micrografías a diferente magnificación donde se aprecia la morfología característica de Hap sintetizada con 0.3 \%wt de PVP.
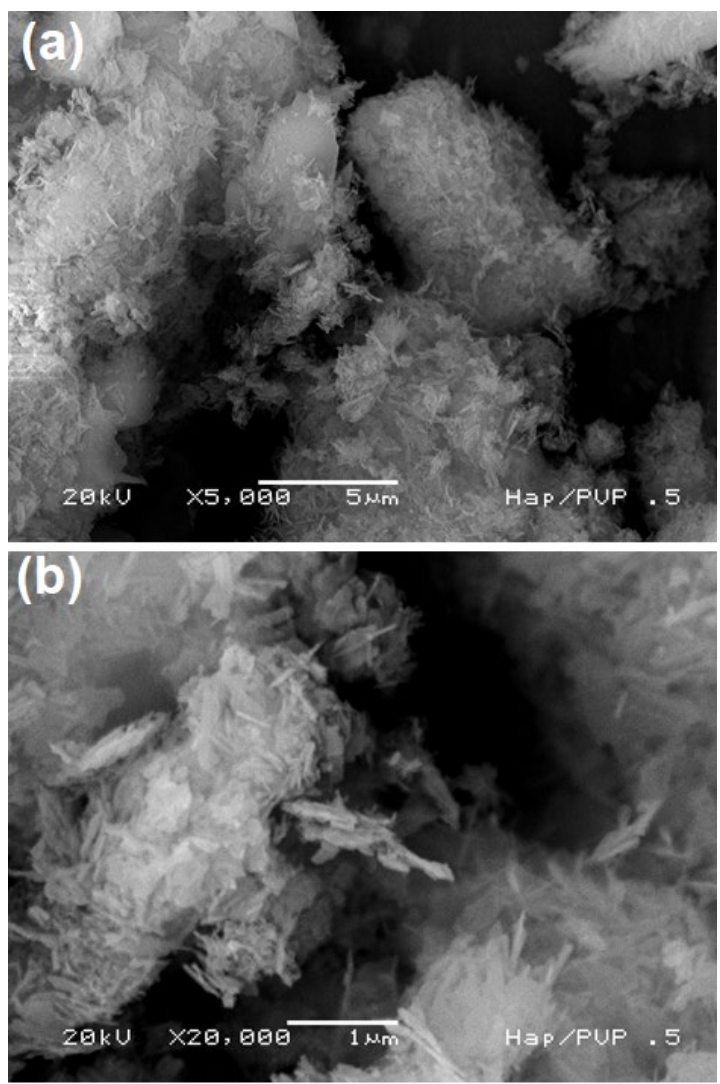

Figura 7. Micrografías a diferente magnificación donde se aprecia la morfología característica de Hap sintetizada con 0.3 \%wt de PVP.
La Tabla 1 presenta la composición elemental para la Hap pura y sintetizada en presencia de PVP. En ésta se nota la presencia de calcio $(\mathrm{Ca})$, fósforo $(\mathrm{P})$, oxígeno $(\mathrm{O})$ para la Hap pura, en tanto que el carbono (C) también estuvo presente, en mínimas cantidades, en las muestras sintetizadas en presencia de PVP. En el último renglón se incluye la relación porcentual en peso (\%wt) $\mathrm{Ca} / \mathrm{P}$, teniéndose el valor más alto $(2.13)$ para la Hap pura y el valor más bajo para la Hap con $0.1 \%$ wt de PVP (1.50); tomando en cuenta que la relación estequiométrica $\mathrm{Ca} / \mathrm{P}$ es de $1.67(\approx 2.16$ $\%$ wt) (Ramesh, 2007), la muestra que más se aproxima a este valor corresponde a la Hap sintetizada en ausencia de PVP.

Tabla 1. Composición elemental para la Hap pura y sintetizada en presencia de diferentes porcentajes de PVP.

\begin{tabular}{ccrrc}
\hline Elemento & Pura & $0.1 \%$ wt & $0.3 \%$ wt & $0.5 \%$ wt \\
\hline $\mathrm{C}$ & 0 & 0.10 & 0.40 & 1.04 \\
$\mathrm{O}$ & 31.07 & 40.94 & 38.30 & 37.35 \\
$\mathrm{P}$ & 21.99 & 23.57 & 22.72 & 22.34 \\
$\mathrm{Ca}$ & 46.94 & 35.43 & 38.58 & 39.27 \\
$\mathrm{Ca} / \mathrm{P}$ & 2.13 & 1.50 & 1.69 & 1.75 \\
\hline
\end{tabular}

\section{Discusión}

La microscopia electrónica de barrido permitió visualizar la modificación de la morfología (forma y tamaño) de la Hap conforme aumenta la concentración porcentual de PVP. Los resultados aquí presentados indican que para bajas concentraciones $(0$ y $0.1 \% \mathrm{wt})$ se forman nanopartículas con aparente forma esférica con diámetros menores a $100 \mathrm{~nm}$; por otro lado, al aumentar la concentración de PVP ( 0.3 y $0.5 \%$ wt) se aprecia el crecimiento de estructuras tipo rods con ancho nanométrico y longitud o largo que puede ser mayor a los micrómetros; A.J. Nathanael y colaboradores también utilizaron el PVP en la síntesis de Hap reportando, de manera similar, la formación de estructuras nanométricas tipo rods debido a la presencia de este polímero (Nathanael, 2015). Haber logrado sintetizar estructuras tipo rods en este trabajo es destacable tomando en cuenta que desde hace algunas décadas se hacen esfuerzos para sintetizar nuevas estructuras y estudiar como las variaciones de tamaño y morfología afecta la respuesta ya sea como biomaterial, como material óptico, catalizador, etc.; en el caso de los rods, la relación largo-ancho es fundamental en éstas propiedades.

El uso de polímeros como el PVP para controlar el crecimiento de las nanopartículas ha sido constantemente reportado ya que por sus propiedades químicas, se puede adsorber en la superficie de las nanopartículas inhibiendo de esta manera el proceso de crecimiento en todas las direcciones, solo facilitando el crecimiento en una sola dirección (Figura 8); este es debido a las propiedades anfifilicas del PVP, donde un extremo de la molécula es hidrofóbica, en tanto que el extremo contrario es hidrofílica.

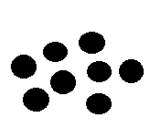

(a)

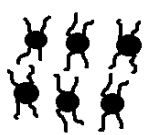

(b)

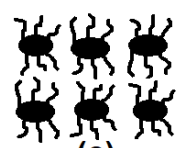

(c)

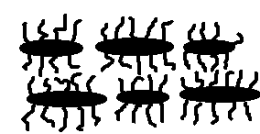

(d)
Figura 8. Esquema de modificación de la morfología de la Hap en presencia de la concentración de PVP, (a) 0, (b) 0.1, (c) 0.3 y (d) $0.5 \%$ wt. 
El análisis por EDS mostró la presencia de $\mathrm{O}, \mathrm{Ca}$ y $\mathrm{P}$ como constituyentes principales en las diferentes Hap sintetizadas, con una relación $\mathrm{Ca} / \mathrm{P}$ en el rango de 1.50 a 2.13 , estando más próxima a la relación estequimétrica la de $0.3 \%$ wt de PVP; en tanto que la presencia de $\mathrm{C}$ se asocia con el PVP adicionado durante la síntesis. Haciendo uso del mapeo se pudo corroborar que la distribución de los elementos identificados es homogénea, tal y como puede apreciarse en el mapeo de la Hap pura en la Figura 9 y el correspondiente para la Hap sintetizada con $0.1 \%$ wt de PVP de la Figura 10.

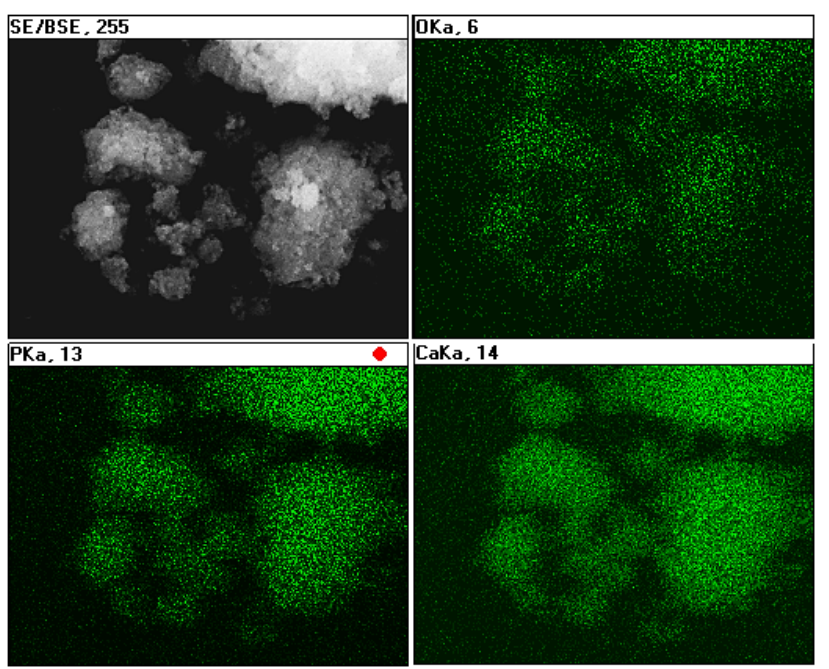

Figura 9. Mapeo elemental por EDS de la muestra de Hap pura. Se observa una distribución homogénea del $\mathrm{O}, \mathrm{P}$ y $\mathrm{Ca}$ en la muestra.
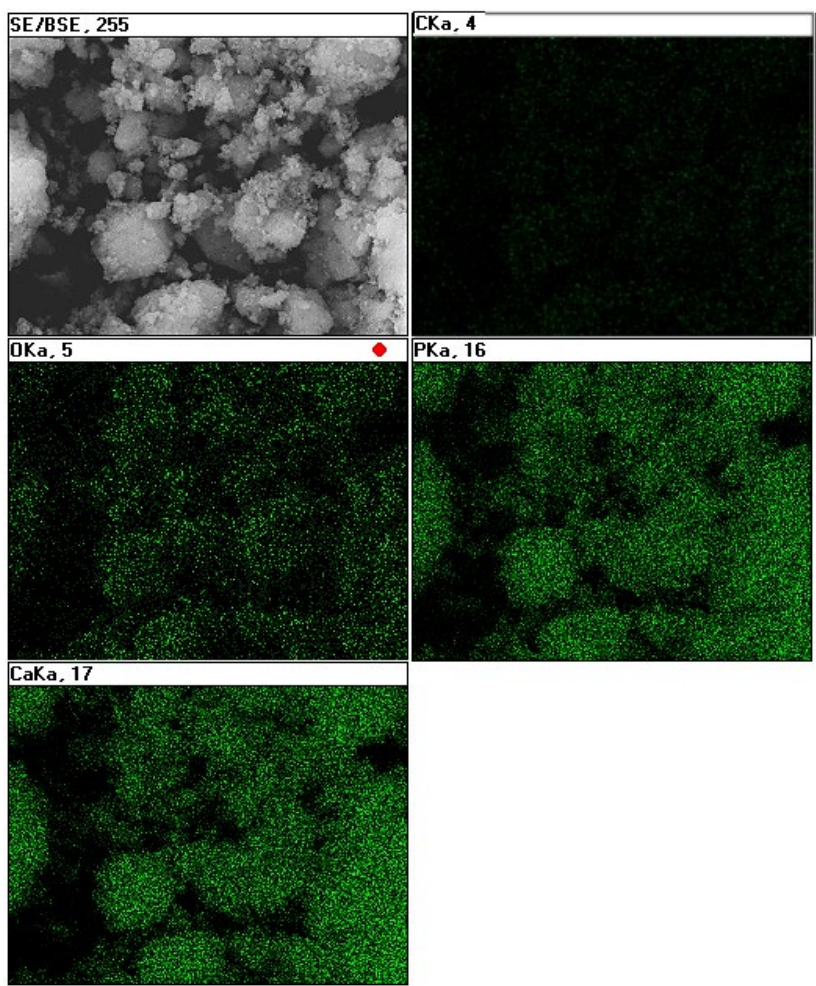

Figura 10. Mapeo elemental por EDS de la muestra de Hap sintetizada en presencia de $0.1 \%$ wt de PVP.

\section{Conclusiones}

A través de MEB-EDS fue posible investigar el efecto del PVP como controlador de la morfología (forma y tamaño) de la hidroxiapatita. Se demostró que la Hap pura consiste en nanopartículas con tendencia esférica y que al aumentar la concentración de PVP, se comienzan a formar estructuras tipo rods cuya longitud se aumenta al incrementar el porcentaje de PVP. Asimismo, el EDS mostró la presencia de oxígeno, calcio y fósforo, principalmente, los cuales están distribuidos de manera homogénea en las muestras analizadas. Estos resultados demuestran que el PVP puede ser utilizado como controlador del crecimiento de la Hap, haciendo posible el uso de este biocerámico para resolver problemas específicos.

\section{Agradecimientos}

Agradecimiento al CONACYT por el apoyo a Lesly Sabina Villaseñor Cerón por la beca otorgada en el doctorado (0432) perteneciente al Área Académica de Ciencias de la Tierra y Materiales e Instituto de Ciencias Básicas e Ingeniería de la Universidad Autónoma del Estado de Hidalgo.

\section{Referencias}

Cerón, L.S.V.; Lugo, V.R.; Alatorre, J.A.A.; Fernández-Garcia, M.E.; ReyesValderrama, M.I.; González-Martínez, P.; Anaya, D.M (2019). Characterization of hap nanostructures doped with $\mathrm{AgNp}$ and the gamma radiation effects. Results Phys., 15, 102702.

Gła, M., Sonia Kudłacik-Kramarczyk, Anna Drabczyk, Janusz Walter, Aleksandra Kordyka, Marcin Godzierz, Rafał Bogucki, Bo'zena Tyliszczak and Agnieszka Sobczak-Kupiec (2021). Hydroxyapatite Obtained via the Wet Precipitation Method and PVP/PVA Matrix as Components of Polymer-Ceramic Composites for Biomedical $\begin{array}{llll}\text { Applications. } & \text { Molecules } & \text { 26, } & \end{array}$ https://doi.org/10.3390/molecules 26144268

González Mancera, Guillermina y Ma. Eugenia Noguez Amaya (2006), Principios de Microscopía Electrónica de Barrido y Microanálisis por Rayos X Característicos. Primera Edición, D.R.C Facultad de Química, UNAM, Ciudad Universitaria, 04510, México, D.F. Impreso y hecho en México, ISBN 970-32-4011-9.

Ipohorski, Miguel y Patricia B. Bozzano (2013). Microscopia Electrónica de Barrido en la Caracterización de Materiales. Ciencia e Investigación Tomo $63 \mathrm{~N}^{\circ} 3$.

Kallum M. Koczkur, Stefanos Mourdikoudis, Lakshminarayana Polavarapu and Sara E. Skrabalak (2015). Polyvinylpyrrolidone (PVP) in nanoparticle synthesis. Dalton Trans., 44, 17883-17905.

Ledea Lozano Oscar E., Haney Castro Fernández, Ramón González Santos, Marcos Farina de Souza, André Linhares Rossi, y Mair de Oliveira (2010). Aplicación de la Microscopia Electrónica de Barrido en la Caracterización de la Poliapatita ${ }^{\circledR}$. Revista CENIC. Ciencias Químicas, vol. 41, pp. 1-6.

Li. J., Koji Inukai, Yosuke Takahashi, Woosuck Shin (2016). Synthesis and size control of monodispersed BaTiO3-PVP nanoparticles. Journal of Asian Ceramic Societies 4, 394-402

López-Ortiz, S.; Mendoza-Anaya, D.; Sánchez-Campos, D.; FernandezGarcía, M.E.; Salinas-Rodríguez, E.; Reyes-Valderrama, M.I.; Rodríguez-Lugo, V. (2020). The pH Effect on the Growth of Hexagonal and Monoclinic Hydroxyapatite Synthesized by the Hydrothermal Method. J. Nanomater.

Mohammed A., Avin Abdullah (2018). Scanning Electron Microscopy (SEM): A Review. Proceedings of 2018 International Conference on Hydraulics and Pneumatics - HERVEX November 7-9, Băile Govora, Romania.

Montes de Oca Valdés, P., Claudia E. Gutiérrez Wing, Pedro R. González Martínez, Federico García Sántibañez-Sánchez, Gerardo Villa-Sánchez, Demetrio Mendoza-Anaya (2019). Las escamas de pescado como materia prima para el desarrollo de sensores de radiación. Publicación Semestral Pädi Vol. 7 No. Especial 2, 104-108

Nathanael, A.J., Young Ho Seo, and Tae Hwan Oh (2015). PVP Assisted Synthesis of Hydroxyapatite Nanorods with Tunable Aspect Ratio and 
Bioactivity. Journal of Nanomaterials, Vol. 2015, Article ID 621785, 9 pages, http://dx.doi.org/10.1155/2015/621785

Ramesh S., C. Y. Tan, M. Hamdi, I. Sopyan, W. D. Teng (2007). The influence of $\mathrm{Ca} / \mathrm{P}$ ratio on the properties of hydroxyapatite bioceramics. Proc. of SPIE Vol. 6423, 64233A. doi: 10.1117/12.779890.

Ramírez-Meneses Esther, Philippot Karine, Chaudret Bruno (2015), Influence of the Reaction Media on the Stabilization of Rhodium Nanostructures, Ingeniería Investigación y Tecnología, volumen XVI (No. 2): 225-237 ISSN 1405-7743 FI-UNAM.

Rodríguez-Lugo, V.; Karthik, T.V.K.; Mendoza-Anaya, D.; Rubio-Rosas, E.; Cerón, L.S.V.; Reyes-Valderrama, M.I.; Salinas-Rodríguez, E. (2018). Wet chemical synthesis of nanocrystalline hydroxyapatite flakes: Effect of $\mathrm{pH}$ and sintering temperature on structural and morphological properties. R. Soc. Open Sci. 5.

Rodríguez-Lugo, V, D. Salado-Leza, S. López-Ortiz, D. Mendoza-Anaya, L. S. Villaseñor-Cerón, M. I. Reyes-Valderrama (2020). Revisión de la Hidroxiapatita Nanoestructurada como Alternativa para Tratamiento de Cáncer. Publicación Semestral Pädi Vol. 8 No. Especial 115-127.

Sánchez-Campos, D.; Mendoza-Anaya, D.; Reyes-Valderrama, M.I.; EstebanGómez, S.; Rodríguez-Lugo, V. (2020). Cationic surfactant at high pH in microwave HAp synthesis. Mater. Lett., 265, 3-6.
Sánchez-Campos, Daniel, Maria Isabel Reyes Valderrama, Susana LópezOrtíz, Daniela Salado-Leza, María Eufemia Fernández-García, Demetrio Mendoza-Anaya, Eleazar Salinas-Rodríguez and Ventura RodríguezLugo (2021). Modulated Monoclinic Hydroxyapatite: The Effect of $\mathrm{pH}$ in the Microwave Assisted Method. Minerals, 11, 314. https://doi.org/10.3390/min11030314

Vernon-Parry K D. (2000). Scanning Electron Microscopy: an introduction Ill-Vs Review, Vol.13 No.4.

Villaseñór Cerón, L.S., D. Mendoza-Anaya, M.I. Reyes-Valderrama, E. Salinas-Rodríguez, V. Rodríguez-Lugo (2019). Estudio termodinámico en la obtención de nanohidroxiapatita por el método de microondas. Publicación Semestral Pädi No. 13, 35-40.

Wang, P.; Li, C.; Gong, H.; Jiang, X.; Wang, H.; Li, K (2010). Effects of synthesis conditions on the morphology of hydroxyapatite nanoparticles produced by wet chemical process. Powder Technol., 203, 315-321.

Zhang, X.; Vecchio, K.S. (2007). Hydrothermal synthesis of hydroxyapatite rods. J. Cryst. Growth, 308, 133-140. 